\title{
Clinical assessment of hepatic de novo lipogenesis in non-alcoholic fatty liver disease
}

\author{
Sabina Paglialunga ${ }^{1 *}$ and Clayton A. Dehn ${ }^{1,2}$
}

\begin{abstract}
Non-alcoholic fatty liver disease (NAFLD) is heralded as the next big global epidemic. Hepatic de novo lipogenesis (DNL), the synthesis of new fatty acids from non-lipid sources, is thought to play a pivotal role in the development of NAFLD. While there is currently no NAFLD-specific therapeutic agent available, pharmaceutical drugs aimed at reducing hepatic fat accretion may prove to be a powerful ally in the treatment and management of this disease. With a focus on NAFLD, the present review summarizes current techniques examining DNL from a clinical perspective, and describes the merits and limitations of three commonly used assays; stable-label isotope tracer studies, fatty acid indexes and indirect calorimetry as non-invasive measures of hepatic DNL. Finally, the application of DNL assessments in the pharmacological and nutraceutical treatment of NAFLD/NASH is summarized. In a clinical research setting, measures of DNL are an important marker in the development of anti-NAFLD treatments.
\end{abstract}

Keywords: Stable label isotope, MIDA, Fatty acid indexes, Indirect calorimetry, Fructose

\section{Background}

In both developed and developing countries, the incidence of metabolic diseases are on the rise and are largely associated with sedentary lifestyles as well as easy access to calorie rich - nutrient low foods. Further, approximately one billion people worldwide are affected by non-alcoholic fatty liver disease (NAFLD) [1], a chronic metabolic derangement caused by an excess of lipid storage within the liver referred to as steatosis, of which obesity and type 2 diabetes are frequent comorbidities [2]. Individuals with increased liver fat content are diagnosed with NAFLD when lipid accumulation in the liver is not related to excessive alcohol intake or viral infection such as hepatitis. NAFLD is typically asymptomatic and may even be benign, but in approximately $20 \%$ of affected individuals prolonged steatosis will lead to a severe form of the disease called non-alcoholic steatohepatitis (NASH) marked by liver inflammation and fibrosis [3]. NASH may further precipitate cirrhosis and even hepatocellular carcinoma

\footnotetext{
* Correspondence: sabina.paglialunga@celerion.com

'Global Clinical Research, Celerion, 2420 West Baseline Road, Tempe, AZ 85283, USA

Full list of author information is available at the end of the article
}

[2]. Excessive dietary intake of saturated fatty acids, increased free-fatty acids derived from lipolysis of white adipose tissue, reduced $\beta$-oxidation within the liver and upregulated hepatic de novo lipogenesis (DNL), the synthesis of new fatty acids from carbohydrates or proteins, are all thought to contribute to liver steatosis $[4,5]$. Furthermore, conditions of insulin resistance and oxidative stress induce a lipotoxic environment within the liver provoking inflammation and hepatocyte injury which can lead to the development of fibrosis (Fig. 1).

There are currently no NAFLD-specific medications available on the market, and a clear pathway to approval is yet to be adopted. The unpredictable and slowly progressive nature of the condition makes the assessment of direct endpoints such as cirrhosis or mortality impractical. As such, the Food and Drug Administration (FDA) is considering the use of a surrogate outcome measures including the reversal of steatohepatitis without the progression of advanced fibrosis to examine the treatment response to novel therapies [6]. Hepatic lipid accretion is implicated in the development and progression of NAFLD (reviewed in $[7,8]$ ), and clinical methods of examining the same are valuable signals for evaluating anti-NAFLD drug efficacy. The present review will 


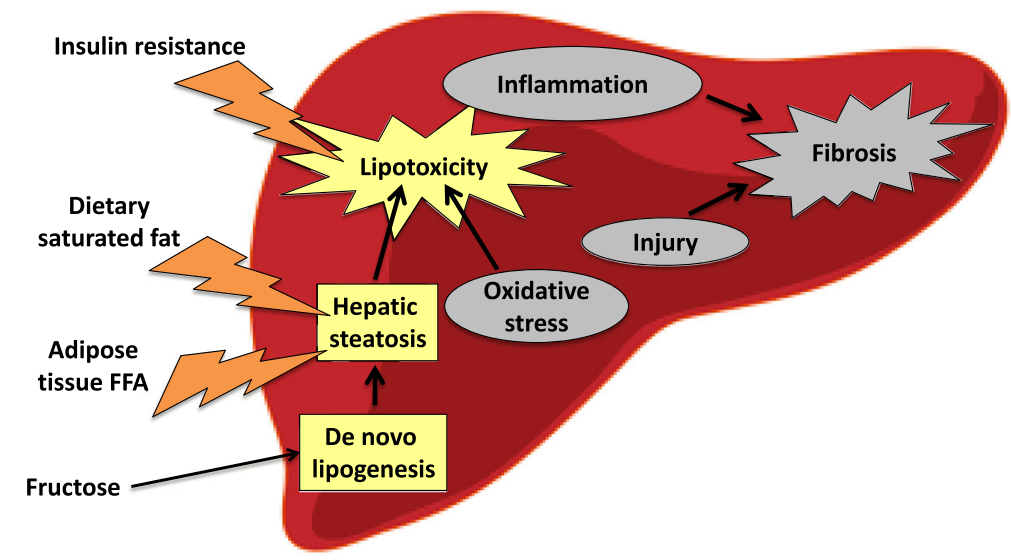

Fig. 1 The pathophysiology of NAFLD/NASH. Excess dietary saturated fat intake as well as increased adipose tissue lipolysis contributes to fat accumulation in the liver. Overconsumption of fructose leading to upregulated de novo lipogenesis (DNL) also adds to hepatic steatosis. Insulin resistance and oxidative stress fuel a lipotoxic environment within the liver, which can result in hepatic inflammation and injury leading to fibrosis

highlight current and novel tools and techniques assessing DNL, and how this analysis is clinically applicable to evaluate the treatment of NAFLD.

\section{De novo fatty acid synthesis}

DNL is a highly regulated pathway, dependent upon several steps, in which key enzymes involved are upregulated in NAFLD $[9,10]$. The process begins with acetyl-CoA, derived from non-lipid sources, as a building block for fatty acid synthesis. The first committed step in this pathway is the formation of malonyl-CoA from acetyl-CoA (Fig. 2). This reaction is catalyzed by acetyl-CoA carboxylase (ACC), of which there are two isoforms (ACC1 and ACC2) (reviewed in [11]). These

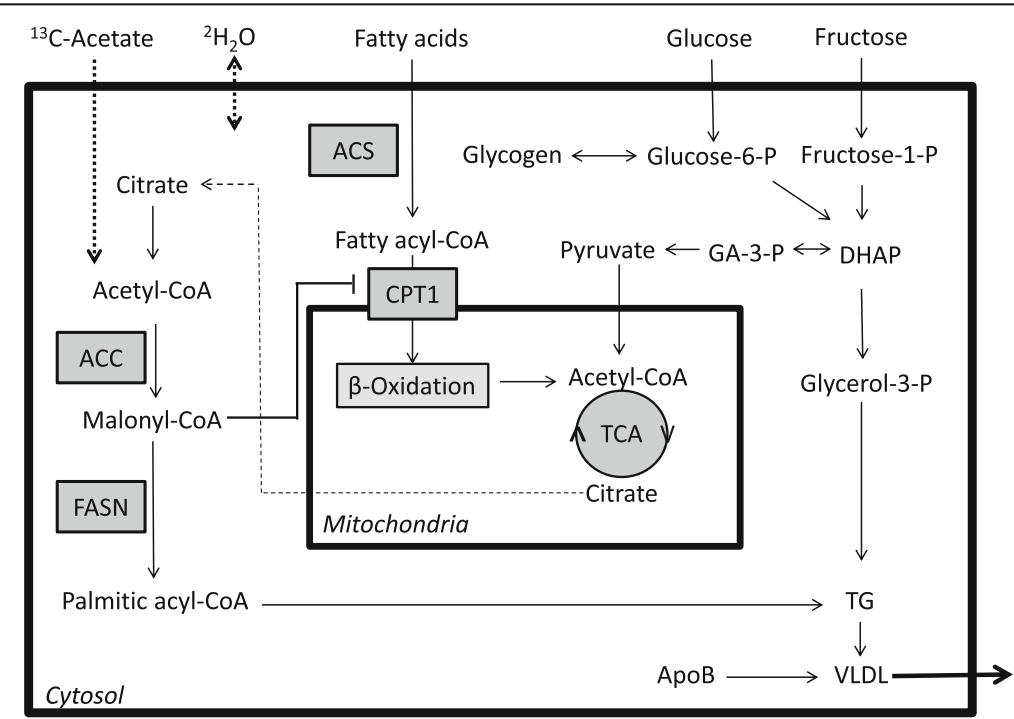

Fig. 2 Hepatic de novo lipogenesis and TG secretion. The DNL process begins with the conversion of acetyl-CoA into malonyl-CoA by ACC. Malonyl-CoA is condensed with several acetyl-CoA moieties by FASN to produce a 16-carbon palmitic acyl-CoA. Three fatty acyl-CoAs are bound to a glycerol backbone to form one TG. Glucose and fructose are taken up by the hepatocyte and may be metabolized to pyruvate for energy formation in the mitochondrial or to glycerol-3-phospate for TG synthesis. Glucose can also be stored as glycogen within the liver cell. TG are combined with apoB and packaged into VLDL for secretion into the blood stream. Malonyl-CoA, also inhibits CPT1, suppressing fatty acid uptake into the mitochondria and $\beta$-oxidation. Dietary fatty acids are also taken up by the hepatocyte and converted to fatty acyl-CoA which can be oxidized or used for TG synthesis (not shown). Stable isotope labels such as ${ }^{13} \mathrm{C}$-acetate and ${ }^{2} \mathrm{H}_{2} \mathrm{O}$ are used to determine the rate of DNL through the incorporation into VLDL-palmitate. ACC, acetyl-CoA carboxylase; ACS, acetyl-CoA synthase; ApoB, apolipoprotein B; CPT1, carnitine palmitoyl transferase 1; DHAP, dihydroxyacetone phosphate; FASN, fatty acid synthase; GA-3-P, glyceraldehyde-3-phosphate; P, phosphate; TCA, the citric acid cycle; TG, triglyceride; VLDL, very low density lipoprotein 
enzymes are derived from independent genes and differ in their tissue distribution and subcellular localization. ACC1 is found in lipogenic tissue and localized in the cytosol, while ACC2 is highly expressed in skeletal and heart muscle and bound to mitochondria [12]. ACC is active in unphosphorylated states that occur during fed and insulin-stimulated conditions resulting in increasing levels of malonyl-CoA. Conversely, ACC is phosphorylated and inactivated by AMPK, interrupting the production of malonyl-CoA. Malonyl-CoA is sequestered towards DNL as a substrate required for fatty acid synthesis and it also directly regulates fatty acid oxidation as an allosteric inhibitor of carnitine palmitoyl transferase 1 (CPT1) [13, 14] (Fig. 2). CPT1 transports longchain fatty acids into the mitochondria as the obligatory step in fatty acid oxidation. The final key regulator of DNL is fatty acid synthase (FASN). This is a multicomplexed enzyme that is responsible for the building of new fatty acids (Fig. 3). The process begins with the condensation of malonyl-CoA onto an acyl-carrier protein (ACP) group, followed by the extension of the malonylACP with an acetyl-CoA subgroup. The resulting acetoacetate moiety is reduced, dehydrated, and reduced again to generate butyryl-ACP. Butyryl-ACP also under goes a round of dehydration and reduction steps and this process repeats six times to produce a 16-carbon long saturated fatty acid called palmitate. FASN is highly regulated at the transcriptional and translational level; its activity is stimulated by insulin and citrate, and inhibited by PKA, AMP and palmitoyl-CoA (review in [15]). The de novo fatty acids produced have multiple fates. They are used for triglyceride (TG) synthesis, oxidized through $\beta$-oxidation, and may function as intracellular signals including acting as PPAR ligands, LXR modulators, lipokines, and substrates for protein palmitoylation (reviewed in [16]).

\section{Hepatic DNL in health and disease}

Early studies suggested hepatic DNL was not a significant in vivo contributor to fat metabolism. These initial findings were, however, likely related to the test subjects and conditions examined. In healthy lean, fasting individuals it was originally estimated that DNL contributed to $<5 \%$ of total TG synthesis [17-19]. However, under fed conditions DNL rates are $\sim 25 \%$ greater than fasting levels [20-24]. In addition, hepatic lipogenesis is 2-fold higher in the elderly compared to young subjects [25]. Moreover, DNL rates compared to healthy controls are upregulated approximately 5-fold in individuals with metabolic disorders such obesity [21, 24] and type 2 diabetes [23, 26]. Although the numbers of studies performed to date are limited [20, 27-29], DNL rates in NAFLD patients have reported an increase of up to 3fold compared to BMI-matched controls [27]. Interestingly, NAFLD is more prominent in men than women [30], suggesting the potential of a protective effect of estrogen on the DNL pathway; Pramflak et al. have recently demonstrated this sexual dimorphism is related to intrahepatic fatty acid partitioning and DNL rates [31]. Augmented hepatic DNL rates are now considered a causal factor in the development of NAFLD [5, 20, 27]. Supporting this, FASN expression and serum content are upregulated in fatty liver disease $[9,10,32]$.

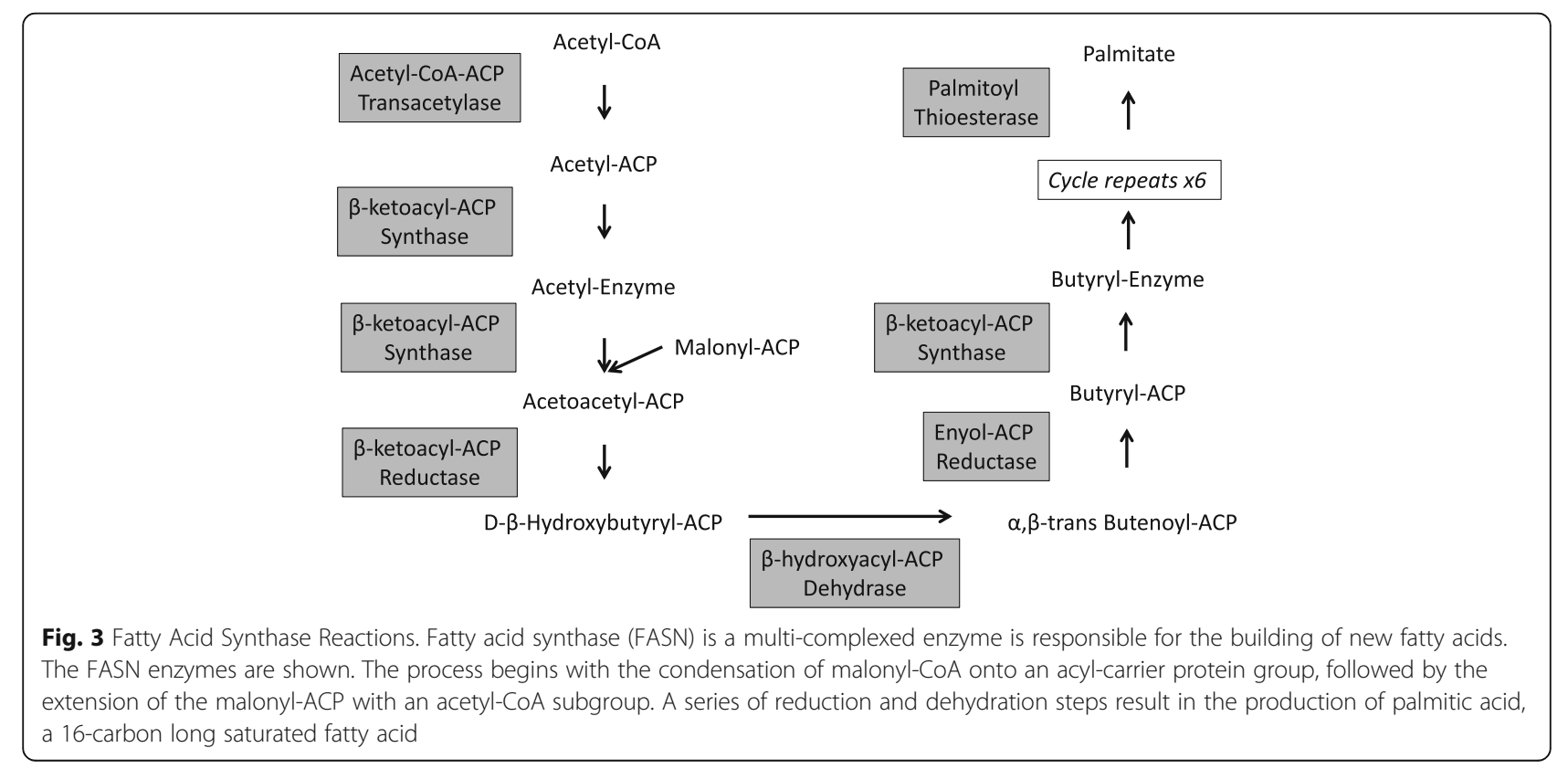




\section{Dietary influences on hepatic lipogenesis}

DNL rates will increase in the fed state with a macronutrient-balanced eucaloric diet. Increasing the percentage intake of carbohydrate will, however, dramatically augment both fasted and fed fractional lipogenesis (reviewed in [17]). As discussed in more detail below, fructose-sweeting beverages are associated with increased liver fat and fractional lipogenesis rates [33, 34], and as a result have been implicated as a contributing factor to NAFLD development [35]. On the other hand, a high starch meal reduces DNL rates [18]. Moreover, diets high in saturated long-chain fatty acids seem to have little impact on hepatic DNL [36]. Furthermore, fish oil supplementation which can lower fructoseinduced hypertriglyceridemia, also showed no effect on DNL rates [37]. There are conflicting animal results regarding the role of medium-chain fatty acids on FASN activity and DNL [38-40], making interpretation difficult. Further, there is little research done to date how the consumption of medium- or short-chain lipids influences hepatic DNL in humans. In addition to macronutrient content, another factor effecting lipogenic response may be meal composition itself. Some studies $[22,41]$, but not all [42] have shown an effect of meal administration on DNL. Oral administration of a test meal results in greater lipogenic rate than duodenal feeding tube, due to small insulin response and slow nutrient delivery associated with the latter [22, 41]. Furthermore, placement of a feeding tube may elicit stress that can dramatically influence fatty acid fluxes. Overall, attention is required to ensure subjects maintain their normal diet or a eucaloric diet prior to study participation and any test meals should be orally consumed where applicable.

\section{Fructose is a lipogenic stimulant}

Since DNL is upregulated in the fed state, administering a test meal can drive fatty acid synthesis rates. A carbohydrate-rich meal (80\% carbohydrate) given as a single bolus can increase DNL rates 3-times over fasting values [21]. Moreover, the type of carbohydrate supplied will influence the net increase. When glucose alone was compared to 50:50 or 25:75 glucose/fructose liquid meals, the presence of fructose significantly augmented DNL rates $[43,44]$. Furthermore, fructose-induced lipogenic response increases in a dose-dependent manner [44]. This is a noteworthy finding since fructose overfeeding is strongly associated with fatty liver disease [33, 34]. In addition, Stanhope et al. found that after 10 weeks of fructose or glucose sweetened-beverage intake, DNL increased from $11 \%$ to $17 \%$ with fructose consumption only [45]. Moreover, a high fructose diet increased fasting DNL from $2 \%$ to $9 \%$ [37]. Fructose preferentially enhances hepatic lipogenesis over glucose since it initially metabolized by fructokinase which is not rate limiting and subsequently broken-down into 3-carbon molecules used for TG synthesis (Fig. 2), and is a potent stimulator of transcription factors regulating lipogenesis (reviewed in [34, 35]). Additionally, fructose feeding results in smaller glycemic excursions and lower insulin response compared to glucose [46], driving substrates towards lipogenesis. The mechanisms of fructoseinduced DNL have been thoroughly describes elsewhere $[33,35]$. From a clinical perspective, employing fructose as a challenge agent for DNL assessment is a potent stimulator of fatty acid synthesis, examining the system's maximum capacity. Furthermore, fructose administration during tracer studies is an effective manner to evaluate the efficacy of a therapeutic intervention on liver fat metabolism [47].

\section{Measuring hepatic DNL in clinical research}

Accessing liver biopsy material for DNL assessment can be risky and difficult, therefore a number of alternative non-invasive approaches have been developed to gain insight into fatty acid synthesis rates in humans. In a clinical setting, DNL can be determined by stable isotope tracer studies, fatty acid profiling or indirect calorimetry; the merits and limitations of each technique are described in Table 1.

\section{Stable isotope tracer studies}

Stable isotope tracers are safe for human use and are widely employed to examine lipid fluxes, turnover rates, lipoprotein production and secretion as well as DNL [48]. In addition, this technique can be readily combined with other measures of metabolism such as clamps, indirect calorimetry or MRS measurements [21, 49]. Moreover, stable isotope DNL measurements are highly reproducible [43]. Newly synthesized fatty acids, as well as those coming from the diet are packaged into TG and secreted from the liver as very low density lipoprotein (VLDL)-TG (Fig. 2). A recent study by Poulsen et al. eloquently demonstrated increased VLDL-TG secretion rates in NAFLD men under fasting as well as insulin stimulated conditions [50]. Hepatic DNL rates can be determined by tracking the VLDL-TG fraction following administration of a labeled substrate. VLDL-TG serves as a marker for assessing the hepatic TG pool. Serum lipoprotein fractions are isolated through ultracentrifugation. Some studies examine DNL rates in the TG-rich lipoproteins (TRL) fraction [43, 46], which is typically identified by a Svedberg flotation rate (Sf) of 60-400, however this fraction may contain both liver and intestinal DNL lipids, therefore it does not reflect a purely hepatic rate. The lipoprotein fraction can be further identified through immunoaffinity chromatograph by apolipoprotein detection for the VLDL-TG fraction. For 
Table 1 Advantages and disadvantages of clinical DNL techniques

\begin{tabular}{lll}
\hline DNL Technique & Advantages & Disadvantages \\
\hline Stable label isotope & Fluxed based assay & Cost of stable label substrates \\
& Selection of stable isotopes & Moderate sensitivity \\
& Reproducible results & Requires specialized equipment for analysis \\
Fatty acid profiling & Relatively inexpensive & \\
& Simple to execute & \\
& High sensitivity & Requires specialized equipment for analysis \\
Indirect calorimetry & High throughput & \\
\hline
\end{tabular}

analysis, lipids are then converted to fatty acid methylesters (FAME) before processing via gas chromatograph mass spectroscopy (GC-MS). Moreover, while most studies measure label-incorporation into palmitic acid $(16: 0)$, this is not a true estimate of total \%DNL as oleic (18:1n9) and myristic (14:0) acids are also major fatty acids produced by FASN [26]. Therefore, lipoprotein fraction (TRL vs. VLDL-TG) and lipid species measured are key methodological considerations that may influence data interpretation and results.

\section{Mass Isotopomer Distribution Analysis (MIDA)}

To determine biosynthesis rates, the enrichment or specific activity of the precursor substrate is required. Since obtaining biopsy material is risky, not always feasible, and is subjected to collection site bias; MIDA was developed to overcome these issues. MIDA estimates the amount of stable isotope in a given tissue like the liver; this analysis was first introduced and applied to lipogenesis studies by Hellerstein and Neese [51]. It is based on the combinatorial probability of polymers repeating from a monomer. The local enriched precursor (e.g. acetylCoA) is determined by the relative distribution pattern of labeled isotopomer in the product (e.g. VLDLpalmitate). In the case of palmitate, the isotopomers could contain anywhere from zero to eight labeled monomers. The fractional abundance of the unlabeled end-product $\left(M_{0}\right)$, and those moieties containing one $\left(M_{1}\right)$, two $\left(M_{2}\right)$ or more $\left(M_{n}\right)$ labeled acetate subunits are measured at baseline and in enriched states, then the fractional rate of lipogenesis is calculated from the rate at which enrichment increases in the product. Before MIDA, errors in estimating biosynthesis rates were unavoidable due to the uncertainty of cytosolic dilution of the precursor substrate [52].

\section{Selecting a stable isotope substrate}

Typically either stable labeled ${ }^{13} \mathrm{C}$-acetate or deuterated water $\left({ }^{2} \mathrm{H}_{2} \mathrm{O}\right)$ are employed for the DNL isotope technique, and the ideal substrate chosen for a particular study depends on the method of administration (iv. infusion vs. consumption), length of isotope priming, and complexity of the study, for example if multiple stable isotopes are used. These considerations are addressed below.

\section{${ }^{13} \mathrm{C}$-acetate}

This tracer is a commonly used substrate in current DNL studies. In the body, acetate is converted to acetylCoA which is the precursor substrate of fatty acid synthesis. Infusion with either $\left[1-{ }^{13} \mathrm{C}\right]$-acetate or $\left[2-{ }^{13} \mathrm{C}\right]-$ acetate show similar results [42], however many researchers have adopted $\left[1-{ }^{13} \mathrm{C}\right]$-acetate as a standard precursor. ${ }^{13} \mathrm{C}$-acetate administration does require a lengthy intravenous infusion time prior to testing. Studies have shown that increasing the infusion time results in a greater $\% \mathrm{DNL}$ rate; typically fractional rates are $<5 \%$ with a short infusion of 8 hours and increases to $>3$-fold after a 24- $\mathrm{h}$ dose in a similar cohort [18, 41]. As a result, the increase of acetate tracer into the precursor pool is in large quantities (several grams). However, longer infusion times are required to overcome a delay in VLDL-TG appearance due to entry into a storage pool [23]. Although extremely rare, there exists a possibility that an acetate precursor may have two labeled carbons - one in the defined position (for example 1-C), and the other carbon labeled occurring due to natural abundance, which would thus be termed $M_{2}$. When combined with other acetates in a fatty acid chain the frequency of $M_{2}$ and $M_{3}$ lipids would increase and the occurrence of $M_{O}$ and $M_{1}$ would be reduced, affecting the calculated rate. However, this is solved by expressing the excess isotopomeric distribution $\left(E M_{2} / E M_{1}\right.$; natural abundance corrected) rather than the absolute isotopomer frequencies $M_{2} / M_{1}$ [42].

\section{Deuterated water}

The labeled hydrogen atoms in deuterated water $\left({ }^{2} \mathrm{H}_{2} \mathrm{O}\right)$ can be incorporated into the fatty acid acyl chain through several means; through a number of water requiring pathways, such as glycolysis and TCA cycle, by direct hydrogen-exchange or, obtained from NADPH as a reducing equivalent for FASN. Furthermore, the proportion of NADPH hydrogen atoms deriving from water will depend on the route of NADPH formation; for example via the pentose pathway, malic enzyme or other 
enzymatic routes. While earlier studies dosed deuterated water for 48 -h prior to testing resulting in low DNL rates (reviewed in [53]), more recent studies have administered heavy water up to 1 week prior to the investigation test day allowing for maximal capture of DNL rates. Typically, a diluted $\left(70 \%{ }^{2} \mathrm{H}_{2} \mathrm{O}\right)$ priming dose is administered followed by several maintenance doses [25-27, 49]. Administration of the label is given orally which is an advantage over the stress that may be associated with lengthy ${ }^{13} \mathrm{C}$-acetate infusion studies. Also, the isotope is relatively inexpensive. Deuterated water is also a preferred stable isotope substrate when other ${ }^{13} \mathrm{C}$-labels substrates are being used, or if the study procedures do not accommodate an IV infusion. On the other hand, there are several shortcomings associated with deuterated water DNL-measurements. In early research, administration of deuterated water induced vertigo [54]. However, improvements to mass spectroscopy instrument sensitivity have prevented the need of heavy water doses large enough to provoke this side-effect. Other limitations include the assumption that plasma ${ }^{2} \mathrm{H}$ enrichment is the same as tissue intracellular pools, moreover the ratio of ${ }^{2} \mathrm{H}: \mathrm{H}$ may not be consistent over various metabolic conditions [55]. In addition, an isotope bias may also exist influencing \%DNL rates. DeuteratedDNL rates were originally reported as parts per thousand change in sample enrichment relative to natural abundance (standard mean ocean water), and used literature based hydrogen/carbon incorporation ratios to estimate synthesis rates [55], which are impacted by several errors and assumptions [56]. However, a variation of MIDA has been utilized for determining the \%DNL with deuterated water as a precursor [57], reconciling previous limitations.

\section{${ }^{13} \mathrm{C}$-glucose and ${ }^{13} \mathrm{C}$-fructose}

Labeled fructose and glucose have been examined as potential DNL tracers however, these are not consider suitable substrates to determine fatty acid synthesis rates. Chong and colleagues demonstrated, approximately $38 \%$ of the label was identified in TG-glycerol fraction, and only minimal amounts $(\sim 0.5 \%)$ were found within TG-fatty acids [46]. Although administration these labels most closely resembles real life situation such as the consumption of a sweetened beverage, this method does not convey the total \%DNL since the majority of the labeled sugars were found to be oxidized [46].

\section{${ }^{13}$ C-ethanol}

Excessive alcohol consumption is often associated with hepatosteatosis, and even acute alcohol intake can upregulate fractional DNL up to $30 \%$ [58]. Since ethanol is converted to acetate (a substrate for FASN) by acetyl$\mathrm{CoA}$ synthetase, ${ }^{13} \mathrm{C}$-ethanol was examined as a potential tracer for DNL assays. Siler et al. found that approximately $70-80 \%$ of the labeled ethanol was found in plasma as enriched acetate, yet $<5 \%$ of the label was identified in VLDL-palmitate [58]. Therefore, while alcohol stimulates hepatic lipid accretion, this is not a direct effect through the provision of acetate-loading in an acute setting and therefore not ideal for experimental purposes. Furthermore, during a clinical study, subjects should be instructed to avoid alcoholic beverage prior to testing due to its stimulatory effect on the DNL pathway.

\section{Fatty acid profiling}

Lipidomics is the study of lipid species and their biological roles, and this emerging field has advanced medical research. Often lipids are altered in a diseased state and novel lipid biomarkers can be useful for diagnosis and evaluating progression and treatment response to a disease [59]. Improvements in sample preparation resulting in greater recovery and efficiency as well as the ability to detect low abundance lipids have significantly contributed to this growing field. For blood samples, mass spectrometry (MS) coupled with chromatography separation is the most widely used technique for lipid quantification, due to the high sensitivity and throughput of this method. Either liquid chromatography (LC) or GC can be integrated for lipidomic studies, however GC-MS is preferred for fatty acid profiling since it is a more rapid and sensitive method. GC tends to yield higher peak resolution than LC. In this respect, fatty acid profiling and lipid indexes are a highly sensitive, alternative approach to tracer studies to examine DNL in a clinical setting (reviewed in [33]). The synthesis of these de novo fatty acids is shown in Fig. 4.

\section{Profiling de novo fatty acids}

Increased DNL has been described as a key player in the development of hepatic lipid accretion and NAFLD. This was elegantly shown by Puri et al., through fatty acid profiling in both plasma and liver biopsy samples from NAFLD patients $[60,61]$. NAFLD subjects demonstrated an increase in de novo lipid species composition, as well as relative and absolute amounts of fatty acids such as palmitic, palmitoleic (16:1n7), stearic (18:0) and oleic (18:1n9) acid. In support of these findings, Lee et al. observed elevated plasma palmitoleic acid (16:1n7) levels in MRS-confirmed fatty liver subjects compared to low fat liver controls [62]. In addition, palmitoleic acid (16:1n7) levels significantly correlated with isotope DNL assessments. More recently, hepatic oleic acid (18:1n9) concentrations determined by ${ }^{1} \mathrm{H}-\mathrm{NMR}$ spectroscopy was found to significantly associate with histology quantified steatosis in NAFLD patients undergoing bariatric surgery [63]. 


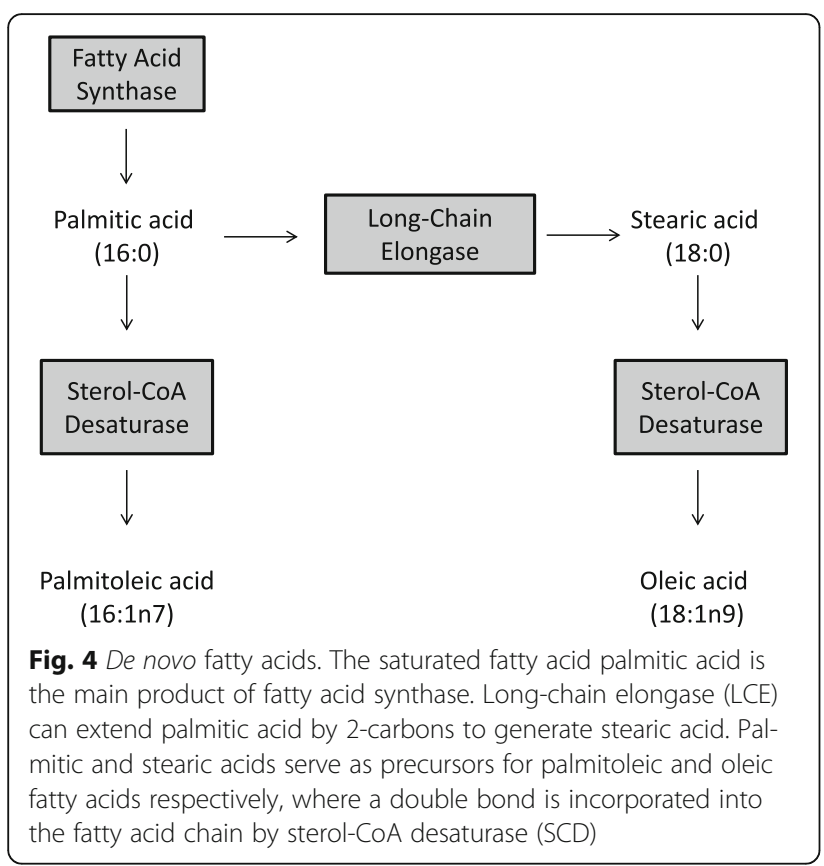

\section{Fatty acid indexes}

In animal models [64] as well as human subjects [60, 61, 63], hepatic steatosis is consistently observed with an attenuation of the polyunsaturated to monounsaturated fatty acids ratio, therefore a number of lipid ratios and indexes have been developed as a surrogate marker of DNL. The lipogenic index introduced by Hudgins et al. in 1996, calculates the ratio of palmitic acid (16:0) to the essential omega-6 linoleic acid (18:2n6), reflecting DNL rates when dietary fats were matched for adipose tissue fatty acid composition [65]. More recently, Chong et al. used this method to evaluate lipogenesis after the consumption of a 3-day high carbohydrate diet [66]. Indeed, the lipogenic index was higher after high carbohydrate feeding compared to a high fat diet. This index has been also analyzed in erythrocyte membranes in the EPIC-Potsdam Study and found to positively correlate with proxies of liver fat content [67]. Other DNL indices include the desaturation index, measuring the ratio of oleic acid (18:1n9) to stearic acid (18:0). Although tested in dogs, this index did not change over an 8-h postprandial phase following a high sucrose meal [68]. However when comparing the TG and phospholipid desaturation index, Peter et al. did find an association with liver fat content in humans with NAFLD [69]. Finally, examining the percent increase in VLDLpalmitate may be a significant biomarker of $\mathrm{DNL}$, as the percent change strongly correlated with the change in $\% \mathrm{DNL}$ fractional rate determined by MIDA over an 8-h postprandial period in response to a fructose challenge [44]. Taken altogether, fatty acid indexes are an effective method for DNL examination and may be considered a suitable alternative to tracer studies. However due to the limited number of studies performed to date in humans; caution is advised when applying this measurement to evaluate the effect of DNL in response to a therapeutic treatment.

\section{Indirect calorimetry}

The DNL reaction is energy demanding, requiring 14 $\mathrm{NADPH}$ resulting in the production of $7 \mathrm{CO}_{2}$ for the generation of 1 palmitate fatty acid. In this respect, indirect calorimetry, a powerful technique used to examine substrate utilization and energy expenditure, has been also widely used as a method to determine wholebody DNL in vivo. Through gas exchange, the respiratory quotient $(\mathrm{RQ})$; the ratio of carbon dioxide production to oxygen consumption, can be calculated. RQ $>1.0$ indicates a net conversion of carbohydrate to lipid. One caveat of this measurement is that it cannot ascertain hepatic-specific DNL per se, as only net whole-body DNL is measured, however, it has been suggested that short-term, postprandial values of $R Q>1.0$ would likely reflect a prominent hepatic contribution [21]. Moreover, this technique may be better suited for some groups over others. For instance, after a carbohydrate-rich meal, net lipogenesis was significantly greater in obese men compared to lean controls [21], however in overweight men and women this trend was not observed [70]. On the other hand, several researchers have found that the RQ does not reach over the lipogenesis threshold when examining carbohydrate-induced DNL in healthy lean populations, as excess dietary carbohydrates were shunted towards glycogen storage [19, 71]. Only after glycogen storage capacity is saturated, increases in DNL can be observed in normal healthy populations through indirect calorimetry. In general, this can be achieved with an extreme dietary intervention such as massive carbohydrate overfeeding, increasing glycogen by $\sim 500 \mathrm{~g}$ [72]. Therefore, as a complementary tool, indirect calorimetry can provide valuable clinical information regarding whole-body metabolism such as fuel selection and energy expenditure which would ultimately impact liver function, however there are many shortcomings associated with using this technique solely for hepatic DNL determinations.

\section{The role of DNL assessment in evaluating NAFLD treatment}

There is currently no NAFLD/NASH-specific medication available on the market. The unmet needs of those with this metabolic disorder have been addressed with lifestyle modifications for weight loss, vitamin supplements $[2,73]$ or insulin sensitizing therapy such as pioglitazone treatment [74]. While these therapeutic agents have yielded some success [75], concerns of increased cardiovascular risks [76] and undesired side-effects [77- 
Table 2 Summary of NAFLD/NASH clinical trials incorporating stable-label isotope DNL assessments

\begin{tabular}{|c|c|c|c|c|c|c|}
\hline Subjects & Study Type & Intervention & Period & Tracer & Outcome & Ref \\
\hline $\begin{array}{l}\text { Healthy controls } \\
(n=\mathrm{NR})\end{array}$ & $\begin{array}{l}\text { Randomized } \\
\text { double-blind, } \\
\text { placebo-controlled } \\
\text { crossover }\end{array}$ & $\begin{array}{l}\text { Compound } 9(600 \mathrm{mg}) \\
\text { or placebo with one week } \\
\text { washout }\end{array}$ & $\begin{array}{l}\text { Single } \\
\text { dose }\end{array}$ & $\begin{array}{l}{\left[1-{ }^{13} \mathrm{C}\right] \text {-acetate }(9-9.5} \\
\mathrm{mg} / \mathrm{min}) \text { for } 20.5 \mathrm{~h}\end{array}$ & $\begin{array}{l}\text { Compound } 9 \text { reduced } \\
\text { fructose-induced } \\
\text { fractional DNL }\end{array}$ & {$[$ [47] } \\
\hline $\begin{array}{l}\text { Type } 2 \text { diabetes } \\
(n=12)\end{array}$ & $\begin{array}{l}\text { Open label, } \\
\text { randomized (1:1) }\end{array}$ & $\begin{array}{l}\text { Pioglitazone (dose } \\
\text { escalated to } 45 \mathrm{mg} / \text { day) } \\
\text { Rosiglitazone (dose escalated } \\
\text { to } 4 \mathrm{mg} \text { twice daily) }\end{array}$ & 20 weeks & $\begin{array}{l}{\left[1-{ }^{13} \mathrm{C}\right] \text {-acetate }(10 \mathrm{mg} / \mathrm{min})} \\
\text { For } 12 \mathrm{~h}\end{array}$ & $\begin{array}{l}\text { Pioglitazone reduced fasting } \\
\text { fractional DNL over course of } \\
\text { acetate infusion } \\
\text { No change with Rosiglitazone }\end{array}$ & [74] \\
\hline $\begin{array}{l}\text { Type } 2 \text { diabetes } \\
(n=60)\end{array}$ & $\begin{array}{l}\text { Randomized } \\
\text { double-blind } \\
\text { placebo-controlled } \\
(1: 1)\end{array}$ & $\begin{array}{l}\text { Colesevelam ( } 3.75 \mathrm{~g} / \text { day) or } \\
\text { placebo }\end{array}$ & 12 weeks & $\begin{array}{l}{\left[1-{ }^{13} \mathrm{C}\right] \text {-acetate (10 mg/min) }} \\
19.5 \mathrm{~h} \text { continuous infusion }\end{array}$ & $\begin{array}{l}\text { Placebo increased fasting and } \\
\text { postprandial fractional DNL, no } \\
\text { change with colesevelam }\end{array}$ & [81] \\
\hline $\begin{array}{l}\text { Biopsy-proven } \\
\text { NASH }(n=14)\end{array}$ & $\begin{array}{l}\text { Randomized } \\
\text { double-blind, } \\
\text { placebo-controlled } \\
(1 ; 1)\end{array}$ & $\begin{array}{l}\text { Liraglutide (dose escalated to } \\
1.8 \mathrm{mg} / \text { day) or placebo }\end{array}$ & 12 weeks & $\begin{array}{l}{ }^{2} \mathrm{H}_{2} \mathrm{O}(3 \mathrm{~g} / \mathrm{kg} \text { total body } \\
\text { water in } 2 \text { doses) plus ad } \\
\text { libitum drinking water } \\
\text { enriched with } \\
0.4 \%{ }^{2} \mathrm{H}_{2} \mathrm{O} \text { over } 20 \mathrm{~h}\end{array}$ & $\begin{array}{l}\text { Liraglutide decreased fasting \% } \\
\text { change DNL }\end{array}$ & [29] \\
\hline
\end{tabular}

NR Not reported

79] remain. Interestingly, pioglitazone treatment, but not rosiglitazone, significantly lowered steady-state fractional DNL in type 2 diabetes subjects with hyperglycemia [80], demonstrating one of pioglitazone's mechanisms as an effective anti-NAFLD agent. In addition to a number of anti-fibrotic and anti-inflammation products under development, drug classes with steatosis lowering capacity are being rigorously investigated for NAFLD/ NASH treatment. However, to our knowledge, only three other published studies to date have evaluated stablelabel isotope DNL response as mode of drug efficacy in the context of fatty liver disease (Table 2). A novel ACC inhibitor (identified as compound 9) dramatically lowered peak fructose-induced fractional DNL by $64 \%$ compared to placebo after a single dose in a phase 1 clinical study [47]. Moreover, the GLP-1 agonist Liragultide reduced change from baseline \%DNL compared to placebo in NASH patients [29]. On the other hand, colesevelam, a bile acid sequestrant, potent cholesterollowering agent and putative anti-NASH agent, showed no effect on hepatic DNL [81]. In addition to pharmacological interventions, a number of nutraceutical products have been shown to display anti-dyslipidemia properties (reviewed in [82]) and even have been evaluated as antiNASH agents [83]. Regarding hepatic DNL, phytochemicals from mulberry leaf and chokeberries as well as other plant sterols have shown promising pre-clinical lowering effects in animal models of NAFLD [84-86] however further studies in humans are needed to evaluate their effectiveness.

\section{Conclusions}

Upregulated DNL is a contributing factor to hepatic steatosis and NAFLD [20, 27, 61]. There are several methodologies to evaluate DNL in a clinical setting. Currently, only a handful of laboratories have the capability and expertise to examine fractional DNL rates with stable isotopes. However, as pharmaceutical and biotech companies develop novel anti-NAFLD drugs that target components of steatosis development such hepatic DNL, it is anticipated that more studies will evaluate DNL through the stable label fractional technique. On the other hand, fatty acid profiling may represent an attractive alternative. There are several limitations associated with indirect calorimetry to measure DNL, however as complementary analysis, this measurement can provide insight into hepatic and whole-body metabolism. In summary, measurements of hepatic lipid accumulation such as DNL assessment either by stable label technique or fatty acid profiling, will become an instrumental marker of drug efficacy for novel NAFLD investigational products and nutraceutical treatment response.

\section{Abbreviations}

ACC: Acetyl-CoA carboxylase; ACP: Acyl-carrier protein; CPT1: Carnitine palmitoyl transferase 1; DNL: De novo lipogenesis; FASN: Fatty acid synthase; FDA: Food and Drug Administration; GC: Gas chromatography; LC: Liquid chromatography; MIDA: Mass isotopomer distribution analysis; MRS: Magnetic resonance spectroscopy; MS: Mass spectrometry; NAFLD: Non-alcoholic fatty liver disease; NASH: Non-alcoholic hepatosteatosis; RQ: Respiratory quotient; TG: Triglycerides; TRL: TG-rich lipoproteins; TZD: Thiazolidinediones; VLDL: Very low density lipoprotein

\section{Acknowledgements}

Not applicable.

\section{Funding}

Not applicable.

\section{Availability of data and material} Not applicable.

\section{Authors' contributions}

SP wrote and edited the manuscript; CAD critically reviewed and edited the manuscript. Both authors read and approved the final manuscript.

Competing interest

SP is employed by Celerion; CAD is the CEO of Umbrella Corporation. 


\section{Consent for publication}

Not applicable.

\section{Ethics approval and consent to participate}

Not applicable.

\section{Author details}

'Global Clinical Research, Celerion, 2420 West Baseline Road, Tempe, AZ 85283, USA. ${ }^{2}$ Current affiliation: Umbrella Corporation, San Antonio, TX, USA

Received: 1 March 2016 Accepted: 30 August 2016

Published online: 17 September 2016

\section{References}

1. Loomba R, Sanyal AJ. The global NAFLD epidemic. Nat Rev Gastroenterol Hepatol. 2013;10:686-90.

2. Chalasani N, Younossi Z, Lavine JE, Diehl AM, Brunt EM, Cusi K, Charlton M Sanyal AJ. The diagnosis and management of non-alcoholic fatty liver disease: practice Guideline by the American Association for the Study of Liver Diseases, American College of Gastroenterology, and the American Gastroenterological Association. Hepatology. 2012;55:2005-23.

3. Wong WW, Wong GL, Choi PC, Chan AW, Li MK, Chan HY, Chim AM, Yu J, Sung JJ, Chan HL. Disease progression of non-alcoholic fatty liver disease: a prospective study with paired liver biopsies at 3 years. Gut. 2010;59:969-74.

4. Cusi K. Role of obesity and lipotoxicity in the development of nonalcoholic steatohepatitis: pathophysiology and clinical implications. Gastroenterology. 2012;142:711-25. e716.

5. Gan L, Xiang W, Xie B, Yu L. Molecular mechanisms of fatty liver in obesity. Front Med. 2015;9:275-87.

6. Paglialunga S, Dehn CA. Follow the yellow brick road: the pathway for NAFLD and NASH drug development in early phase clinical trials. Int J Diabetes and Clin Res. 2015;2:037.

7. Kawano Y, Cohen DE. Mechanisms of hepatic triglyceride accumulation in non-alcoholic fatty liver disease. J Gastroenterol. 2013:48:434-41.

8. Ameer F, Scandiuzzi L, Hasnain S, Kalbacher H, Zaidi N. De novo lipogenesis in health and disease. Metabolism. 2014;63:895-902.

9. Dorn C, Riener MO, Kirovski G, Saugspier M, Steib K, Weiss TS, Gabele E, Kristiansen G, Hartmann A, Hellerbrand C. Expression of fatty acid synthase in nonalcoholic fatty liver disease. Int J Clin Exp Pathol. 2010;3:505-14.

10. Mitsuyoshi H, Yasui K, Harano $Y$, Endo M, Tsuji K, Minami M, Itoh Y, Okanoue T, Yoshikawa T. Analysis of hepatic genes involved in the metabolism of fatty acids and iron in nonalcoholic fatty liver disease. Hepatol Res. 2009;39:366-73.

11. Brownsey RW, Boone AN, Elliott JE, Kulpa JE, Lee WM. Regulation of acetylCoA carboxylase. Biochem Soc Trans. 2006;34:223-7.

12. Abu-Elheiga L, Brinkley WR, Zhong L, Chirala SS, Woldegiorgis G, Wakil SJ. The subcellular localization of acetyl-CoA carboxylase 2. Proc Natl Acad Sci U S A. 2000:97:1444-9.

13. McGarry JD, Foster DW. Hormonal control of ketogenesis. Biochemical considerations. Arch Intern Med. 1977;137:495-501.

14. McGarry JD, Foster DW. Regulation of hepatic fatty acid oxidation and ketone body production. Annu Rev Biochem. 1980;49:395-420.

15. Menendez JA, Vazquez-Martin A, Ortega FJ, Fernandez-Real JM. Fatty acid synthase: association with insulin resistance, type 2 diabetes, and cancer. Clin Chem. 2009:55:425-38.

16. Solinas G, Boren J, Dulloo AG. De novo lipogenesis in metabolic homeostasis: More friend than foe? Mol Metab. 2015:4:367-77.

17. Hellerstein MK. De novo lipogenesis in humans: metabolic and regulatory aspects. Eur J Clin Nutr. 1999:53 Suppl 1:S53-65.

18. Parks EJ, Krauss RM, Christiansen MP, Neese RA, Hellerstein MK. Effects of a low-fat, high-carbohydrate diet on VLDL-triglyceride assembly, production, and clearance. J Clin Invest. 1999;104:1087-96.

19. Schwarz JM, Neese RA, Turner S, Dare D, Hellerstein MK. Short-term alterations in carbohydrate energy intake in humans. Striking effects on hepatic glucose production, de novo lipogenesis, lipolysis, and whole-body fuel selection. J Clin Invest. 1995;96:2735-43.

20. Donnelly KL, Smith Cl, Schwarzenberg SJ, Jessurun J, Boldt MD, Parks EJ. Sources of fatty acids stored in liver and secreted via lipoproteins in patients with nonalcoholic fatty liver disease. J Clin Invest. 2005;115:1343-51.

21. Marques-Lopes I, Ansorena D, Astiasaran I, Forga L, Martinez JA. Postprandial de novo lipogenesis and metabolic changes induced by a high- carbohydrate, low-fat meal in lean and overweight men. Am J Clin Nutr. 2001;73:253-61.

22. Barrows BR, Parks EJ. Contributions of different fatty acid sources to very low-density lipoprotein-triacylglycerol in the fasted and fed states. J Clin Endocrinol Metab. 2006;91:1446-52.

23. Vedala A, Wang W, Neese RA, Christiansen MP, Hellerstein MK. Delayed secretory pathway contributions to VLDL-triglycerides from plasma NEFA, diet, and de novo lipogenesis in humans. J Lipid Res. 2006;47:2562-74.

24. Schwarz JM, Linfoot P, Dare D, Aghajanian K. Hepatic de novo lipogenesis in normoinsulinemic and hyperinsulinemic subjects consuming high-fat, low-carbohydrate and low-fat, high-carbohydrate isoenergetic diets. Am J Clin Nutr. 2003;77:43-50.

25. Flannery C, Dufour S, Rabol R, Shulman Gl, Petersen KF. Skeletal muscle insulin resistance promotes increased hepatic de novo lipogenesis, hyperlipidemia, and hepatic steatosis in the elderly. Diabetes. 2012;61:2711-7.

26. Wilke MS, French MA, Goh YK, Ryan EA, Jones PJ, Clandinin MT. Synthesis of specific fatty acids contributes to VLDL-triacylglycerol composition in humans with and without type 2 diabetes. Diabetologia. 2009;52:1628-37.

27. Lambert JE, Ramos-Roman MA, Browning JD, Parks EJ. Increased de novo lipogenesis is a distinct characteristic of individuals with nonalcoholic fatty liver disease. Gastroenterology. 2014;146:726-35.

28. Diraison F, Moulin P, Beylot M. Contribution of hepatic de novo lipogenesis and reesterification of plasma non esterified fatty acids to plasma triglyceride synthesis during non-alcoholic fatty liver disease. Diabetes Metab. 2003;29:478-85.

29. Armstrong MJ, Hull D, Guo K, Barton D, Hazlehurst JM, Gathercole LL, Nasiri M, Yu J, Gough SC, Newsome PN, Tomlinson JW. Glucagon-like peptide 1 decreases lipotoxicity in non-alcoholic steatohepatitis. J Hepatol. 2016;64: 399-408.

30. Vernon G, Baranova A, Younossi ZM. Systematic review: the epidemiology and natural history of non-alcoholic fatty liver disease and non-alcoholic steatohepatitis in adults. Aliment Pharmacol Ther. 2011:34:274-85.

31. Pramfalk C, Pavlides M, Banerjee R, McNeil CA, Neubauer S, Karpe F, Hodson L. Sex-specific differences in hepatic fat oxidation and synthesis may explain the higher propensity for NAFLD in men. J Clin Endocrinol Metab. 2015; 100(12):4425-33. doi:10.1210/jc.2015-2649.

32. Notarnicola M, Misciagna G, Tutino V, Chiloiro M, Osella AR, Guerra V, Bonfiglio C, Caruso MG. Increased serum levels of lipogenic enzymes in patients with severe liver steatosis. Lipids Health Dis. 2012;11:145.

33. Moore JB, Gunn PJ, Fielding BA. The role of dietary sugars and de novo lipogenesis in non-alcoholic fatty liver disease. Nutrients. 2014;6:5679-703.

34. Nseir W, Nassar F, Assy N. Soft drinks consumption and nonalcoholic fatty liver disease. World J Gastroenterol. 2010;16:2579-88.

35. Softic S, Cohen DE, Kahn CR. Role of Dietary Fructose and Hepatic De Novo Lipogenesis in Fatty Liver Disease. Dig Dis Sci. 2016;61(5):1282-93.

36. Konrad SD, Cook SL, Goh YK, French MA, Clandinin MT. Use of deuterium oxide to measure de novo fatty acid synthesis in normal subjects consuming different dietary fatty acid composition1. Biochim Biophys Acta. 1998;1393: 143-52.

37. Faeh D, Minehira K, Schwarz JM, Periasamy R, Park S, Tappy L. Effect of fructose overfeeding and fish oil administration on hepatic de novo lipogenesis and insulin sensitivity in healthy men. Diabetes. 2005;54:1907-13.

38. Geelen MJ. Medium-chain fatty acids as short-term regulators of hepatic lipogenesis. Biochem J. 1994;302(Pt 1):141-6.

39. Shinohara H, Ogawa A, Kasai M, Aoyama T. Effect of randomly interesterified triacylglycerols containing medium- and long-chain fatty acids on energy expenditure and hepatic fatty acid metabolism in rats. Biosci Biotechnol Biochem. 2005;69:1811-8.

40. Arunima S, Rajamohan T. Influence of virgin coconut oil-enriched diet on the transcriptional regulation of fatty acid synthesis and oxidation in rats - a comparative study. Br J Nutr. 2014;111:1782-90.

41. Timlin MT, Barrows BR, Parks EJ. Increased dietary substrate delivery alters hepatic fatty acid recycling in healthy men. Diabetes. 2005;54:2694-701.

42. Hellerstein MK, Kletke C, Kaempfer S, Wu K, Shackleton CH. Use of mass isotopomer distributions in secreted lipids to sample lipogenic acetyl-CoA pool in vivo in humans. Am J Physiol. 1991;261:E479-86.

43. Parks EJ, Skokan LE, Timlin MT, Dingfelder CS. Dietary sugars stimulate fatty acid synthesis in adults. J Nutr. 2008;138:1039-46.

44. Hudgins LC, Parker TS, Levine DM, Hellerstein MK. A dual sugar challenge test for lipogenic sensitivity to dietary fructose. J Clin Endocrinol Metab. 2011;96:861-8. 
45. Stanhope KL, Schwarz JM, Keim NL, Griffen SC, Bremer AA, Graham JL, Hatcher B, Cox CL, Dyachenko A, Zhang W, et al. Consuming fructosesweetened, not glucose-sweetened, beverages increases visceral adiposity and lipids and decreases insulin sensitivity in overweight/obese humans. J Clin Invest. 2009;119:1322-34.

46. Chong MF, Fielding BA, Frayn KN. Mechanisms for the acute effect of fructose on postprandial lipemia. Am J Clin Nutr. 2007;85:1511-20.

47. Griffith DA, Kung DW, Esler WP, Amor PA, Bagley SW, Beysen C, CarvajalGonzalez S, Doran SD, Limberakis C, Mathiowetz AM, et al. Decreasing the rate of metabolic ketone reduction in the discovery of a clinical acetyl-CoA carboxylase inhibitor for the treatment of diabetes. J Med Chem. 2014;57: 10512-26.

48. Magkos F, Mittendorfer B. Stable isotope-labeled tracers for the investigation of fatty acid and triglyceride metabolism in humans in vivo. Clin Lipidol. 2009;4:215-30.

49. Rabol R, Petersen KF, Dufour S, Flannery C, Shulman Gl. Reversal of muscle insulin resistance with exercise reduces postprandial hepatic de novo lipogenesis in insulin resistant individuals. Proc Natl Acad Sci U S A. 2011;108:13705-9.

50. Poulsen MK, Nellemann B, Stodkilde-Jorgensen H, Pedersen SB, Gronbaek H, Nielsen S. Impaired Insulin Suppression of VLDL-Triglyceride Kinetics in Nonalcoholic Fatty Liver Disease. J Clin Endocrinol Metab. 2016;101(4):1637-46. doi:10.1210/jc.2015-3476

51. Hellerstein MK, Neese RA. Mass isotopomer distribution analysis: a technique for measuring biosynthesis and turnover of polymers. Am J Physiol. 1992;263:E988-E1001.

52. Dietschy JM, Brown MS. Effect of alterations of the specific activity of the intracellular acetyl CoA pool on apparent rates of hepatic cholesterogenesis. J Lipid Res. 1974;15:508-16.

53. Jones PJ. Tracing lipogenesis in humans using deuterated water. Can J Physiol Pharmacol. 1996;74:755-60.

54. Brandt T. Positional and positioning vertigo and nystagmus. J Neurol Sci. 1990;95:3-28.

55. Leitch CA, Jones PJ. Measurement of human lipogenesis using deuterium incorporation. J Lipid Res. 1993;34:157-63.

56. Murphy EJ. Stable isotope methods for the in vivo measurement of lipogenesis and triglyceride metabolism. J Anim Sci. 2006;84(Suppl):E94-E104.

57. Lee WN, Bassilian S, Ajie HO, Schoeller DA, Edmond J, Bergner EA, Byerley LO. In vivo measurement of fatty acids and cholesterol synthesis using D2O and mass isotopomer analysis. Am J Physiol. 1994;266:E699-708.

58. Siler SQ, Neese RA, Hellerstein MK. De novo lipogenesis, lipid kinetics, and whole-body lipid balances in humans after acute alcohol consumption. Am J Clin Nutr. 1999;70:928-36.

59. Yang L, Li M, Shan Y, Shen S, Bai Y, Liu H. Recent advances in lipidomics for disease research. J Sep Sci. 2016;39:38-50.

60. Puri P, Wiest MM, Cheung O, Mirshahi F, Sargeant C, Min HK, Contos MJ, Sterling RK, Fuchs M, Zhou H, et al. The plasma lipidomic signature of nonalcoholic steatohepatitis. Hepatology. 2009;50:1827-38.

61. Puri P, Baillie RA, Wiest MM, Mirshahi F, Choudhury J, Cheung O, Sargeant C, Contos MJ, Sanyal AJ. A lipidomic analysis of nonalcoholic fatty liver disease. Hepatology. 2007;46:1081-90.

62. Lee JJ, Lambert JE, Hovhannisyan Y, Ramos-Roman MA, Trombold JR, Wagner DA, Parks EJ. Palmitoleic acid is elevated in fatty liver disease and reflects hepatic lipogenesis. Am J Clin Nutr. 2015;101:34-43.

63. Calvo N, Beltran-Debon R, Rodriguez-Gallego E, Hernandez-Aguilera A, Guirro M, Marine-Casado R, Milla L, Alegret JM, Sabench F, del Castillo D, et al. Liver fat deposition and mitochondrial dysfunction in morbid obesity: An approach combining metabolomics with liver imaging and histology. World J Gastroenterol. 2015;21:7529-44.

64. Vinaixa M, Rodriguez MA, Rull A, Beltran R, Blade C, Brezmes J, Canellas N, Joven J, Correig X. Metabolomic assessment of the effect of dietary cholesterol in the progressive development of fatty liver disease. J Proteome Res. 2010;9:2527-38.

65. Hudgins LC, Hellerstein M, Seidman C, Neese R, Diakun J, Hirsch J. Human fatty acid synthesis is stimulated by a eucaloric low fat, high carbohydrate diet. J Clin Invest. 1996;97:2081-91.

66. Chong MF, Hodson L, Bickerton AS, Roberts R, Neville M, Karpe F, Frayn KN, Fielding BA. Parallel activation of de novo lipogenesis and stearoyl-CoA desaturase activity after $3 \mathrm{~d}$ of high-carbohydrate feeding. Am J Clin Nutr. 2008;87:817-23.

67. Jacobs $S$, Jager $S$, Jansen $E$, Peter A, Stefan N, Boeing H, Schulze MB, Kroger J. Associations of Erythrocyte Fatty Acids in the De Novo Lipogenesis
Pathway with Proxies of Liver Fat Accumulation in the EPIC-Potsdam Study. PLoS One. 2015;10:e0127368.

68. Harding SV, Bateman KP, Kennedy BP, Rideout TC, Jones PJ. Desaturation index versus isotopically measured de novo lipogenesis as an indicator of acute systemic lipogenesis. BMC Res Notes. 2015;8:49.

69. Peter A, Cegan A, Wagner S, Elcnerova M, Konigsrainer A, Konigsrainer I, Haring HU, Schleicher ED, Stefan N. Relationships between hepatic stearoylCoA desaturase-1 activity and mRNA expression with liver fat content in humans. Am J Physiol Endocrinol Metab. 2011;300:E321-6.

70. Minehira K, Vega N, Vidal H, Acheson K, Tappy L. Effect of carbohydrate overfeeding on whole body macronutrient metabolism and expression of lipogenic enzymes in adipose tissue of lean and overweight humans. Int J Obes Relat Metab Disord. 2004;28:1291-8.

71. Acheson KJ, Flatt JP, Jequier E. Glycogen synthesis versus lipogenesis after a 500 gram carbohydrate meal in man. Metabolism. 1982:31:1234-40.

72. Acheson KJ, Schutz Y, Bessard T, Anantharaman K, Flatt JP, Jequier E. Glycogen storage capacity and de novo lipogenesis during massive carbohydrate overfeeding in man. Am J Clin Nutr. 1988;48:240-7.

73. Sanyal AJ, Friedman SL, McCullough AJ, Dimick-Santos L, American Association for the Study of Liver D, United States F, Drug A. Challenges and opportunities in drug and biomarker development for nonalcoholic steatohepatitis: findings and recommendations from an American Association for the Study of Liver Diseases-U.S. Food and Drug Administration Joint Workshop. Hepatology. 2015;61:1392-405.

74. Cusi K. Treatment of patients with type 2 diabetes and non-alcoholic fatty liver disease: current approaches and future directions. Diabetologia 2016 59:1112-1120. doi:10.1007/s00125-016-3952-1.

75. Musso G, Gambino R, Cassader M, Pagano G. A meta-analysis of randomized trials for the treatment of nonalcoholic fatty liver disease. Hepatology. 2010; 52:79-104.

76. Miller 3rd ER, Pastor-Barriuso R, Dalal D, Riemersma RA, Appel LJ, Guallar E. Meta-analysis: high-dosage vitamin E supplementation may increase allcause mortality. Ann Intern Med. 2005;142:37-46.

77. Turner LW, Nartey D, Stafford RS, Singh S, Alexander GC. Ambulatory treatment of type 2 diabetes in the U.S., 1997-2012. Diabetes Care. 2014;37:985-92.

78. Lewis JD, Ferrara A, Peng T, Hedderson M, Bilker WB, Quesenberry Jr CP, Vaughn DJ, Nessel L, Selby J, Strom BL. Risk of bladder cancer among diabetic patients treated with pioglitazone: interim report of a longitudinal cohort study. Diabetes Care. 2011;34:916-22.

79. Nissen SE, Wolski K. Effect of rosiglitazone on the risk of myocardial infarction and death from cardiovascular causes. N Engl J Med. 2007:356:2457-71.

80. Beysen C, Murphy EJ, Nagaraja H, Decaris M, Riiff T, Fong A, Hellerstein MK, Boyle PJ. A pilot study of the effects of pioglitazone and rosiglitazone on de novo lipogenesis in type 2 diabetes. J Lipid Res. 2008;49:2657-63.

81. Beysen C, Murphy EJ, Deines K, Chan M, Tsang E, Glass A, Turner SM, Protasio J, Riiff T, Hellerstein MK. Effect of bile acid sequestrants on glucose metabolism, hepatic de novo lipogenesis, and cholesterol and bile acid kinetics in type 2 diabetes: a randomised controlled study. Diabetologia. 2012;55:432-42.

82. Scicchitano PCM, Maiello M, Modesti PA, Muiesan ML, Novo S, Palmiero P, Saba PS, Pedrimelli R, Ciccone MM. Nutraceuticals and dyslipidaemia: Beyond the common therapeutics. J Funct Foods. 2014;6:21.

83. Dasarathy S, Dasarathy J, Khiyami A, Yerian L, Hawkins C, Sargent R, McCullough AJ. Double-blind randomized placebo-controlled clinical trial of omega 3 fatty acids for the treatment of diabetic patients with nonalcoholic steatohepatitis. J Clin Gastroenterol. 2015:49:137-44.

84. Ann JY, Eo H, Lim Y. Mulberry leaves (Morus alba L.) ameliorate obesityinduced hepatic lipogenesis, fibrosis, and oxidative stress in high-fat diet-fed mice. Genes Nutr. 2015;10:46.

85. Park H, Liu Y, Kim HS, Shin JH. Chokeberry attenuates the expression of genes related to de novo lipogenesis in the hepatocytes of mice with nonalcoholic fatty liver disease. Nutr Res. 2016;36:57-64.

86. Rideout TC, Harding SV, Jones PJ. Consumption of plant sterols reduces plasma and hepatic triglycerides and modulates the expression of lipid regulatory genes and de novo lipogenesis in C57BL/6 J mice. Mol Nutr Food Res. 2010;54 Suppl 1:S7-S13. 